Pacific Journal of Mathematics

ON ADDITIVE FUNCTIONS WHOSE LIMITING
DISTRIBUTIONS POSSESS A FINITE MEAN AND VARIANCE 


\title{
ON ADDITIVE FUNCTIONS WHOSE LIMITING DISTRIBUTIONS POSSESS A FINITE MEAN AND VARIANCE
}

\author{
P. D. T. A. ELLIOTT
}

In this paper two characterizations are given of those additive arithmetic functions which possess a limiting distribution with a finite mean and variance. It turns out that the study of such functions fits naturally within the framework of the theory of Lambert series.

1. An arithmetic function $f(n)$ is said to be additive if for every pair of coprime positive integers $a$ and $b$ the relation

$$
f(a b)=f(a)+f(b)
$$

is satisfied. If in addition the relations

$$
f(p)=f\left(p^{2}\right)=\cdots
$$

hold for each prime power then we say that $f(n)$ is strongly additive. For clarity of exposition only we shall confine ourselves to the study of strongly additive functions in this paper.

For each real number $x \geqq 1$ we define the frequency function

$$
\nu_{x}(n ; f(n)<z)=x^{-1} \sum_{\substack{n \leq x \\ f(n)<z}} 1 .
$$

If as $x \rightarrow \infty$ these frequencies converge to a limiting distribution in the usual probabilistic sense then we say that $f(n)$ has a limiting distribution.

2. Theorem. For any (real valued) additive function $f(n)$ the following three propositions are equivalent: variance.

(i) $f(n)$ has a limiting distribution with finite mean and

(ii) The series

$$
\sum f(p) p^{-1} \text { and } \sum f^{2}(p) p^{-1}
$$

both converge.

(iii)

$$
\limsup _{x \rightarrow \infty} x^{-1} \sum_{n \leqq x} f^{2}(n)<\infty
$$

and 


$$
\lim _{x \rightarrow \infty} x^{-1} \sum_{n \leq x} f(n)
$$

exist.

Remark. The equivalence of Propositions (i) and (ii) is exactly what one should expect from the interpretation of $f(n)$ as the sum of independent random variables which take (respective) values $f(p)$ with probability $p^{-1}$ and zero with probability $1-p^{-1}$. More surprising, perhaps, is the fact that the hypothesis that $f(n)$ be additive improves the otherwise weak conditions (iii) to equivalence with (i). We shall (perhaps surprisingly) appeal to a result concerning Lambert series.

It will be clear that a form of theorem involving complex-valued additive functions could be proved if we confine our attention to the equivalence of Propositions (ii) and (iii).

3. Proof that (i) implies (ii).

We define the function

$$
f^{1}(p)=\left\{\begin{array}{l}
f(p) \text { if }|f(p)|<1 \\
1 \text { otherwise }
\end{array}\right.
$$

Then the Erdös-Wintner criterion (see for example Kubilius [3] Theorem $4.5 \mathrm{pp}$. 74-85) asserts that $f(n)$ possesses a limiting frequency (unrestricted) if and only if both of the series

$$
\sum f^{\prime}(p) p^{-1} \text { and } \sum\left(f^{\prime}(p)\right)_{p^{-1}}^{2}
$$

converge. Let $F(z)$ denote the limiting frequency guaranteed by (i). Then for any positive real number $B$ such that $\pm B$ are continuity points of $F(z)$ we see that

$$
x^{-1} \sum_{\substack{n \leq x \\ \mid f(n) \leq B}} f^{2}(n) \longrightarrow \int_{|z| \leqq B} z^{2} d F(z), \quad(x \longrightarrow \infty) .
$$

Next, for any real $\varepsilon>0$ there is a number $A$ such that

$$
\liminf _{x \rightarrow \infty} \nu_{x}(n ;|f(n)| \leqq A)>1-\varepsilon .
$$

From the Erdös-Wintner criterion we see that those primes $q_{j}$ for which $\left|f\left(q_{j}\right)\right| \geqq 1$ are such that the series

$$
\sum q_{j}^{-1}
$$

converges. Let us denote the set of these primes by $Q$.

A straightforward application of the sieve of Eratosthenes shows that those integers which are prime to every $q_{j}$ have a natural density. In fact we obtain 


$$
\nu_{x}\left(n ; q_{j} \nmid n \forall j\right) \longrightarrow \prod_{i=1}^{\infty}\left(1-\frac{1}{q_{i}}\right), \quad(x \longrightarrow \infty) \text {. }
$$

Set $\alpha$ for this product, and let $A$ be chosen so that the second of our two assertions above holds with $\varepsilon=\alpha / 2$. Let the integers $n_{i}$ run through all those integers $n$ which satisfy both

$$
|f(n)| \leqq A \text { and } q_{j} \nmid n \forall j \text {. }
$$

From what we have so far said it is clear that

$$
\liminf _{x \rightarrow \infty} \nu_{x}\left(n ; n=n_{i} \leqq x\right) \geqq \alpha / 2,
$$

and in particular we have

$$
\nu_{x}\left(n ; n=n_{i} \leqq x\right) \geqq \alpha / 4
$$

for all $x \geqq x_{0}$, say.

Consider the sum

$$
S_{x}=\sum_{n_{i} q_{j} \leqq x} \sum_{i}^{\prime} f\left(n_{i} q_{j}\right)^{2}
$$

where' denotes that the side condition $2 A<\left|f\left(q_{j}\right)\right| \leqq B-A$ is to be satisfied.

From these restrictions a typical summand satisfies

$$
f^{2}\left(n_{i} q_{j}\right) \geqq\left(\left|f\left(q_{j}\right)\right|-A\right)^{2} \geqq \frac{1}{4} f^{2}\left(q_{j}\right)
$$

so that

$$
\begin{aligned}
S_{x} & \geqq \frac{1}{4} \sum_{q_{j}^{-1} \leqq x} f^{2}\left(q_{j}\right) \sum_{n_{i} \leqq x q-1} 1 \\
& \geqq \frac{1}{4} \sum_{q_{j} \leqq x x_{0}^{-1}} f^{2}\left(q_{j}\right) \frac{1}{4} \alpha \frac{x}{q_{j}}
\end{aligned}
$$

and therefore

$$
\begin{array}{r}
\limsup _{x \rightarrow \infty} \sum_{q_{j} \leqq x} \frac{f^{2}\left(q_{j}\right)}{q_{j}} \leqq \limsup _{x \rightarrow \infty} x^{-1} S_{x} \\
\leqq \int_{|z| \leqq B} z^{2} d F(z) \leqq \int_{-\infty}^{\infty} z^{2} d F(z) .
\end{array}
$$

Since these inequalities hold for any sequence of suitable continuity points $\pm B$ which tend (in absolute value) to infinity, we deduce that for any $B>0, x \geqq 0$

where

$$
\sum_{q_{j} \leqq x} f^{2}\left(q_{j}\right) \leqq \int_{-\infty}^{\infty} z^{2} d F(z)
$$

$$
2 A<\left|f\left(q_{j}\right)\right| \leqq B-A
$$


so that letting $B \rightarrow \infty$ and then $x$ yields

$$
\sum_{\left|f\left(q_{j}\right)\right| \geq 2 A} \frac{f^{2}\left(q_{j}\right)}{q_{j}}<\infty
$$

Moreover,

$$
\sum_{1 \leqq|f(p)| \leqq 2 A} \frac{|f(p)|}{p} \leqq 2 A \sum_{j=1}^{\infty} \frac{1}{q_{j}}<\infty
$$

and

$$
\sum_{|f(p)|<1} \frac{f^{2}(p)}{p}<\infty
$$

so that altogether the series

$$
\sum f^{2}(p) p^{-1}
$$

converges. The convergence of the second series in (ii) follows immediately.

Proof that (ii) implies (iii) and (i).

We begin with the remark that for any additive function, complex valued or otherwise, the Turan-Kubilius inequality (see for example Kubilius [3] pp. 31-35) asserts that for a suitable positive constant $c$

$$
\sum_{n \leqq x}\left|f(n)-\sum_{p \leqq x} f(p) p^{-1}\right|^{2} \leqq c \sum_{p \leqq x}\left|f^{2}(p)\right| p^{-1}, \quad(x \geqq 1) .
$$

In our present circumstances the sums

$$
\sum_{p \leqq x} f(p) p^{-1} \text { and } \sum_{p \leqq x} f^{2}(p) p^{-1}
$$

are uniformly bounded for all real values of $x$, so that

$$
\begin{aligned}
\sum_{n \leqq x} f^{2}(n) & \leqq 2 \sum_{n \leqq x}\left(f(n)-\sum_{p \leqq x} f(p) p^{-1}\right)^{2}+2 x\left(\sum_{p \leqq x} f(p) p^{-1}\right)^{2} \\
& =O(x)
\end{aligned}
$$

and

$$
\limsup _{x \rightarrow \infty} x^{-1} \sum_{n \leqq x} f^{2}(n)=D<\infty .
$$

From the Erdös-Wintner criterion $f(n)$ possesses a limiting distribution $F(z)$, say. For each real number $B$ such that $\pm B$ are continuity points of this limiting distribution, an application of Fatou's lemma yields

$$
\int_{: z \mid \leqq B} z^{2} d F(z) \leqq \liminf _{x \rightarrow \infty} x^{-1} \sum_{n \leqq x} f^{2}(n) \leqq D
$$


Since $B$ is otherwise arbitrary $F(z)$ has a finite second moment, and hence a finite mean and variance.

This completes the proof of (i).

Furthermore,

$$
x^{-1} \sum_{\substack{n \leq x \\|f(n)| \leq B}} f(n) \longrightarrow \int_{|z| \leqq B} z d F(z), \quad(x \rightarrow \infty),
$$

whilst

$$
\limsup _{x \rightarrow \infty} x^{-1} \sum_{\substack{n \leq x \\|f(n)|>B}}|f(n)| \leqq B^{-1} \limsup _{x \rightarrow \infty} x^{-1} \sum_{n \leqq x} f^{2}(n) \leqq B^{-1} D
$$

from which it follows trivially that as $x \rightarrow \infty$

$$
x^{-1} \sum_{n \leq x} f(n)
$$

converges to the mean of $F(z)$.

This completes the proof of (iii).

Proof that (iii) implies (ii) (which will complete the proof of the theorem).

As one would expect this part of the proof takes a little more effort since we have to start, so to speak, from scratch. We recall that an additive function $f(n)$ is said to be finitely distributed if and only if there are two positive real numbers $c_{1}$ and $c_{2}$ so that for an unbound sequence of real numbers $x \geqq 1$ we can find at least $k \geqq c_{2} x$ integers $1 \leqq a_{1}<a_{2}<\cdots<a_{k} \leqq x$ so that

$$
\left|f\left(a_{i}\right)-f\left(a_{j}\right)\right| \leqq c_{1}
$$

holds for every pair $\left(a_{i}, a_{j}\right), 1 \leqq i, j \leqq k$. This concept was introduced by Erdös [1] who proved

LEMma 1. A function $f(n)$ is finitely distributed if and only if there is a constant $c_{3}$ and an additive function $g(n)$ so that

$$
f(n)=c_{3} \log n+g(n),
$$

where

$$
\sum\left(g^{\prime}(p)\right)^{2} p^{-1}<\infty
$$

There is an alternative proof, on somewhat different lines, given by Ryavec [4].

In our present circumstances we have 


$$
x^{-1} \sum_{n \leqq x} f^{2}(n) \leqq E
$$

for all $x \geqq 2$ (say). Thus for any positive real number $A>E^{1 / 2}$,

$$
\nu_{x}(n ;|f(n)| \geqq A) \leqq E A^{-2}<1, \quad(x \geqq 2) .
$$

It follows from Lemma 1 that $f(n)$ is finitely distributed, and has the form

$$
c_{3} \log n+g(n) \text {. }
$$

Let $\pi$ denote the set of primes $q_{j}$ on which $\left|g\left(q_{j}\right)\right|>A$. Let $n_{i}$ run through those squarefree integers which are prime to each $q_{j}$. Since

$$
\sum_{q \in \pi} q^{-1}
$$

converges, a straightforward application of the sieve of Eratosthenes shows that

$$
\nu_{x}\left(n ; n=n_{i} \leqq x\right) \longrightarrow \prod_{i=1}^{\infty}\left(1-\frac{1}{q_{i}+1}\right)=\beta>0, \quad(x \longrightarrow \infty),
$$

say. For each integer $n$ let $\nu(n)$ denote the number of distinct prime divisors of $n$. We next assume that $c_{3} \neq 0$ and obtain a contradiction.

Let $c_{4}$ be sufficiently large that the inequality $A \nu(n) \leqq c_{4} \log n$ holds for all integers $n \geqq 2$. Then for every real number $x \geqq 2$ we have

$$
\begin{aligned}
E x & \geqq \sum_{i \leqq x} f^{2}\left(n_{i}\right) \geqq \sum_{n_{i} \leqq x}\left(c_{3} \log n_{i}-A \nu\left(n_{i}\right)\right)^{2} \\
& =c_{3}^{2} \sum_{n_{i} \leq x} \log ^{2} n_{i}+O\left(\log x \sum_{n \leqq x} \nu(n)\right) .
\end{aligned}
$$

For all sufficiently large values of $x$ the first of these two terms is

$$
(1+o(1)) \beta c_{3}^{2} x \log ^{2} x
$$

whilst the second is at most $O(x \log x \log \log x)$. This clearly yields a contradiction. Hence $c_{3}=0$ and the additive function $f(n)$ satisfies

$$
\sum_{p}\left(f^{\prime}(p)\right)^{2} p^{-1}<\infty
$$

We now argue exactly as in the proof that the existence of a limiting distribution for $f(n)$ which has a finite variance implies that the series

$$
\sum f^{2}(p) p^{-1}
$$

converges, and deduce the same result.

It remains to secure the convergence of the series 


$$
\sum f(p) p^{-1}
$$

(We do not as yet know that a limiting distribution for $f(n)$ exists, although if we set $\alpha_{n}=\sum_{p \leqq n} f^{\prime}(p) p^{-1}$ then we do know that $f(n)-\alpha_{n}$ has a limiting distribution. See, for example, Kubilius [3] Theorem 4.4 pp. 72-74.)

Consider the generating function

$$
G(z)=\sum_{n=1}^{\infty} f(n) z^{n}
$$

If $N$ is any positive integer and $z$ is any complex number then by the Cauchy-Schwarz inequality

$$
\begin{aligned}
\left|\sum_{N<n \leqq 2 N} f(n) z^{n}\right|^{2} & \leqq \sum_{n \leqq 2 N} f^{2}(n) \sum_{N<n \leqq 2 N}|z|^{2 n} \\
& \leqq E N^{2}|z|^{2 N} .
\end{aligned}
$$

It is easily seen that $G(z)$ is defined by an absolutely convergent series if $z$ satisfies $|z|<1$. By means of the representation

$$
f(n)=\sum_{p \mid n} f(p)
$$

we invert the order of summation to obtain:

$$
G(z)=\sum_{p} f(p) \frac{z^{p}}{1-z^{p}}
$$

Since

$$
x^{-1} \sum_{n \leq x} f(n) \longrightarrow A,(x \longrightarrow \infty), \text { say },
$$

it is readily established that for real values of $z$

$$
G(z) \sim \frac{A}{1-z} \text { as } z \longrightarrow 1-.
$$

We now appeal to a Tauberian theorem concerning Lambert series.

Lemma 2. Let $a_{n} n=1,2, \cdots$ be a series of real numbers, and define

$$
H(y)=\sum_{n=1}^{\infty} a_{n} \frac{n y e^{-n y}}{1-e^{-n y}}
$$

for positive real values of $y$. Let $H(y) \rightarrow A$ as $y \rightarrow 0+$. Let the sum of the $a_{n}$ be a slowly decreasing function in the sense of Hardy [2] $\S 6.2 \mathrm{pp} .124-125$, that is if $x<y$ are real numbers, so that as $x \rightarrow \infty$ and $y \rightarrow \infty$ in such a manner that $y / x \rightarrow 1$, then 


$$
\liminf _{x \rightarrow \infty} \sum_{x<n \leq y} a_{n} \geqq 0
$$

Then

$$
\sum_{n \leqq x} a_{n} \longrightarrow A, \quad(x \longrightarrow \infty) .
$$

REMARK. If the $a_{n}$ are allowed to be complex then provided that we replace the condition of slowly decreasing by a condition of slow oscillation viz:

$$
\lim _{x \rightarrow \infty} \sum_{x<n \leqq y} a_{n}=0,
$$

the same conclusion may be drawn. A proof of this lemma can be found in Hardy [2], Theorem 261, pp. 373-374.

In our present circumstances we set

$$
a_{n}=\left\{\begin{array}{l}
f(p) p^{-1} \text { if } n=p, \\
0 \text { otherwise }
\end{array}\right.
$$

and have established that

$$
H(y)=y G\left(e^{-y}\right) \longrightarrow A, \quad(y \longrightarrow 0+) .
$$

Moreover,

$$
\left(\sum_{x<n \leqq 2 x}\left|a_{n}\right|\right)^{2} \leqq \sum_{x<n \leqq 2 x} f^{2}(p) p^{-1} \sum_{x<n \leqq 2 x} p^{-1},
$$

so that since the series $\sum f^{2}(p) p^{-1}$ converges and

$$
\sum_{x<n \leqq 2 x} \frac{1}{p}=\log \left(\frac{\log 2 x}{\log x}\right)+O\left((\log x)^{-1}\right) \leqq c_{4}<\infty,
$$

we see that the condition of slow decreasing required for an application of Lemma 2 is satisfied.

We deduce that

$$
\lim _{x \rightarrow \infty} \sum_{p \leqq x} \frac{f(p)}{p}=A=\lim _{x \rightarrow \infty} x^{-1} \sum_{n \leqq x} f(n) .
$$

Moreover, by (ii) a limiting distribution exists for $f(n)$, which has the finite mean of value $A$.

This completes the proof of the theorem.

REMARK. The use of the Tauberian theorem in Lemma 2 is very convenient for the study of additive functions. If $f(p)$ assumes complex values the side condition $f(p)=O(\log p)$ will suffice in order for Lemma 2 to be applicable. This is a condition which is satisfied in nearly every case of number theoretical interest. 


\section{REFERENCES}

1. P. Erdös, On the distribution of additive functions, Ann. Math., 47 (1946), 1-20.

2. G. H. Hardy, Divergent Series, Oxford, 1949.

3. J. P. Kubilius, Probabilistic Methods in the Theory of Numbers, Amer. Math. Soc. Transl., Vol. 11, 1964.

4. C. A. Ryavec, A characterization of finitely distributed additive functions. J. Number Theory, 2 (4) (1970), 393-403.

Received May 22, 1972.

UNIVERSITY OF COLORADO 



\section{PACIFIC JOURNAL OF MATHEMATICS}

\section{EDITORS}

RICHARD ARENS (Managing Editor)

University of California

Los Angeles, California 90024

R. A. Beaumont

University of Washington

Seattle, Washington 98105
J. DUGUNDJI*

Department of Mathematics

University of Southern California

Los Angeles, California 90007

D. Gilbarg and J. Milgram

Stanford University

Stanford, California 94305

\section{ASSOCIATE EDITORS}
E. F. BECKENBACH
B. H. NeumanN
F. WOLF
K. YosHIDA

\section{SUPPORTING INSTITUTIONS}

UNIVERSITY OF BRITISH COLUMBIA
CALIFORNIA INSTITUTE OF TECHNOLOGY
UNIVERSITY OF CALIFORNIA
MONTANA STATE UNIVERSITY
UNIVERSITY OF NEVADA
NEW MEXICO STATE UNIVERSITY
OREGON STATE UNIVERSITY
UNIVERSITY OF OREGON
OSAKA UNIVERSITY

UNIVERSITY OF BRITISH COLUMBIA

UNIVERSITY OF CALIFORNIA

MONTANA STATE UNIVERSITY

UNIVERSITY OF NEVADA

OREGON STATE UNIVERSITY

OSAKA UNIVERSITY
UNIVERSITY OF SOUTHERN CALIFORNIA

STANFORD UNIVERSITY

UNIVERSITY OF TOKYO

UNIVERSITY OF UTAH

WASHINGTON STATE UNIVERSITY

UNIVERSITY OF WASHINGTON

\section{AMERICAN MATHEMATICAL SOCIETY} NAVAL WEAPONS CENTER

* C. R. DePrima California Institute of Technology, Pasadena, CA 91109, will replace J. Dugundji until August 1974. 


\section{Pacific Journal of Mathematics}

\section{Vol. 48, No. $1 \quad$ March, 1973}

Jan Aarts and David John Lutzer, Pseudo-completeness and the product of Baire

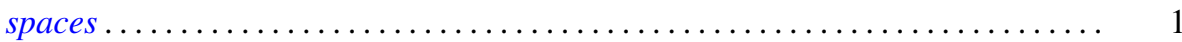

Gordon Owen Berg, Metric characterizations of Euclidean spaces ............ 11

Ajit Kaur Chilana, The space of bounded sequences with the mixed topology ..... . 29

Philip Throop Church and James Timourian, Differentiable open maps of

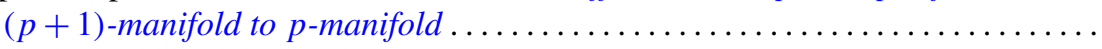

P. D. T. A. Elliott, On additive functions whose limiting distributions possess a finite

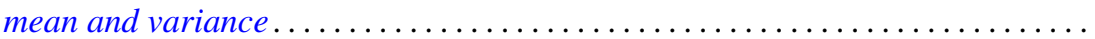

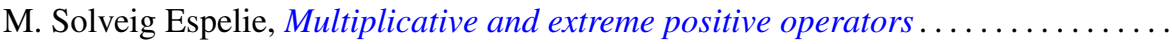

Jacques A. Ferland, Domains of negativity and application to generalized convexity

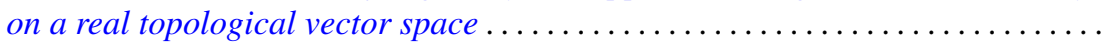

Michael Benton Freeman and Reese Harvey, A compact set that is locally holomorphically convex but not holomorphically convex ...............

Roe William Goodman, Positive-definite distributions and intertwining

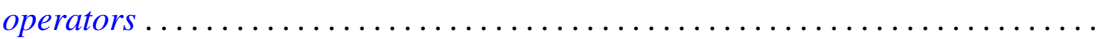

Elliot Charles Gootman, The type of some $C^{*}$ and $W^{*}$-algebras associated with

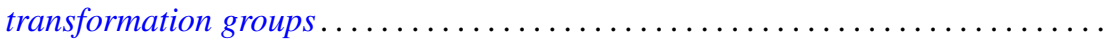

David Charles Haddad, Angular limits of locally finitely valent holomorphic

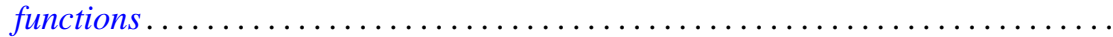

William Buhmann Johnson, On quasi-complements .

William M. Kantor, On 2-transitive collineation groups of finite projective spaces...

Joachim Lambek and Gerhard O. Michler, Completions and classical localizations of right Noetherian rings

Kenneth Lamar Lange, Borel sets of probability measures ......

David Lowell Lovelady, Product integrals for an ordinary differential equation in a Banach space

Jorge Martinez, A hom-functor for lattice-ordered groups .........

W. K. Mason, Weakly almost periodic homeomorphisms of the two sphere ....

Anthony G. Mucci, Limits for martingale-like sequences .......

Eugene Michael Norris, Relationally induced semigroups ...

Arthur E. Olson, A comparison of c-density and $k$-density ......

Donald Steven Passman, On the semisimplicity of group rings of linear groups.

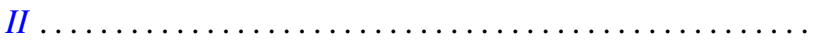

Charles Radin, Ergodicity in von Neumann algebras .

P. Rosenthal, On the singularities of the function generated by the Bergman operator of the second kind.

Arthur Argyle Sagle and J. R. Schumi, Multiplications on homogeneous spaces,

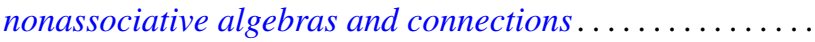

Leo Sario and Cecilia Wang, Existence of Dirichlet finite biharmonic functions on

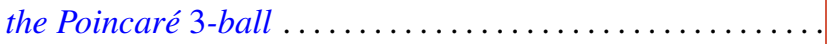

Ramachandran Subramanian, On a generalization of martingales due to Blake ..

Bui An Ton, On strongly nonlinear elliptic variational inequalities.

Seth Warner, A topological characterization of complete, discretely valued

fields. 